\title{
CORRIGENDUM
}

\section{Artificial introgression of a large chromosome fragment around the rice blast resistance gene $P i-t a$ in backcross progeny and several elite rice cultivars}

\author{
Y Jia \\ Molecular Plant Pathology Program, USDA-ARS Dale Bumpers National Rice Research Center, Stuttgart, AR, USA
}

Heredity (2009) 103, 355-356; doi:10.1038/hdy.2009.117

Correction to: Heredity (2009) 103, 333-339; doi:10.1038/ hdy.2009.95

Since the publication of the above paper, the authors have noticed that Figure 3 is incorrect and that there are three type errors.

The correct type is shown with the correct Figure 3.

The authors would like to apologize for any inconvenience caused.

1. In the abstract, 'a temperate japonica cultivar M202 (Pi-ta)' should be 'a temperate japonica cultivar M202 (pi-ta)'.
2. In Materials and methods and Acknowledgements, 'Harold Rockleman' should be spelled as 'Harold Bockleman'.

3. In Materials and methods, 'a total of 50 resistant $\mathrm{BC}_{5} \mathrm{~F}_{1}$ were identified (Table 1)' should be 'a total of 27 resistant $\mathrm{BC}_{5} \mathrm{~F}_{1}$ were identified (Table 1)'.

'Genotype of rice cultivar IR64 between SSR markers RM7102 and RM511 is not the same as that of Tetep and Taducan'. The correct data were presented in the Supplementary materials. 


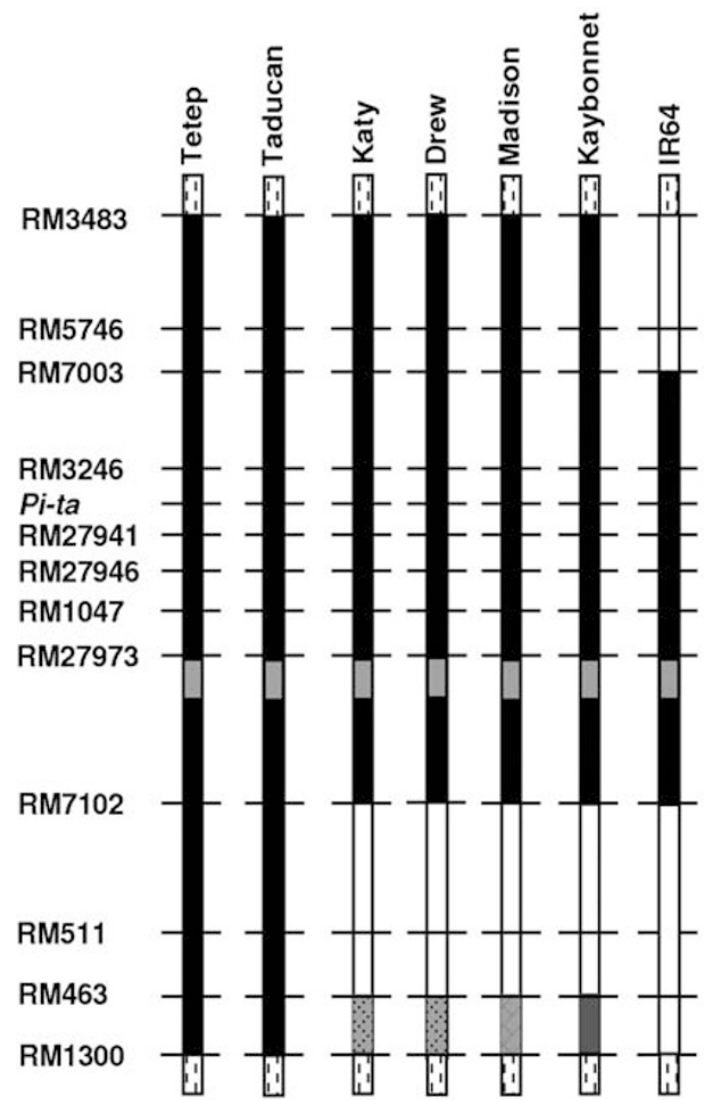

Figure 3 Graphic genotypes of Pi-ta containing landraces and cultivars. Approximate location of SSR markers on Figure 2 was shown. Tetep allele was shown as dark-shaded region and non-Tetep is shown in the white region. Unknown genotypes at both ends were indicated with broken lines. Different color shading was used for different genotype. 